\title{
Ginsenoside Rb1 administration attenuates focal cerebral ischemic reperfusion injury through inhibition of HMGB1 and inflammation signals
}

\author{
ANXIN LIU ${ }^{1}$, WEIWEI ZHU ${ }^{2}$, LIRUI SUN ${ }^{3}$, GUANGMING HAN $^{1}$, HUIPING LIU $^{4}$, \\ ZHAOYU CHEN $^{1}$, LI ZHUANG ${ }^{1}$, WEN JIANG ${ }^{5}$ and XIA XUE ${ }^{6}$
}

\begin{abstract}
${ }^{1}$ Healthcare Ward, Rizhao Hospital of TCM, Rizhao, Shandong 276000; ${ }^{2}$ Department of Pharmacy, Yantai Yuhuangding Hospital Affiliated to Qingdao University, Yantai, Shandong 264000; ${ }^{3}$ Department of Pharmacy, The First Hospital of Jilin University, Changchun, Jilin 130021; ${ }^{4}$ Shandong Anzhuo Medical Technology Co. Ltd., Yantai, Shandong 264000; ${ }^{5}$ Central Laboratory, The Second Hospital of Shandong University; ${ }^{6}$ Department of Pharmacy, The Second Hospital of Shandong University, Jinan, Shandong 250033, P.R. China
\end{abstract}

Received August 16, 2017; Accepted June 27, 2018

DOI: $10.3892 /$ etm.2018.6523

\begin{abstract}
High-mobility group box 1 (HMGB1) is released after focal cerebral ischemia/reperfusion (I/R), and aggravates brain tissue damage. Ginsenoside Rb1 (Rb1), isolated from Panax ginseng, has been reported to inhibit I/R-induced cell death in the brain. The present study aimed to investigate the protective ability of GRb1 on focal cerebral I/R rats and to explore its further mechanisms. A middle cerebral artery occlusion (MCAO) rat model was established and treated with different doses of Rb1. The neurological deficits were examined after reperfusion, and TTC staining was applied to assess the infarct volume. Histology and TUNEL staining were performed to evaluate pathological changes and neuronal cell apoptosis in brain tissues. HMGB1 and levels of inflammatory factors and proteins, were examined by ELISA or western blotting. Rb1 treatment notably improved the neurological deficits in an MCAO model, accompanied by decreased infarct volume in the brain tissues. Histological examination revealed that the necrotic tissue area in MCAO rats was also diminished by $\mathrm{Rb} 1$ treatment. Apoptosis induced by cerebral $\mathrm{I} / \mathrm{R}$ was also attenuated by $\mathrm{Rb} 1$ treatment via downregulation of cleaved caspase-3 and caspase-9 levels. HMGB1 release was inhibited by $\mathrm{Rb} 1$ treatment in MCAO rats, and the levels of nuclear factor- $\kappa \mathrm{B}$, tumor necrosis factor- $\alpha$, interleukin- 6 , inducible nitric oxide synthase and nitric oxide were also decreased. The present study suggests that Rbl serves a protective role in I/R-induced cerebral-neuron injury, due to the decreased
\end{abstract}

Correspondence to: Mrs. Xia Xue, Department of Pharmacy, The Second Hospital of Shandong University, 247 Beiyuan Street, Jinan, Shandong 250033, P.R. China

E-mail: xue_1209@hotmail.com

Key words: ginsenoside Rb1, cerebral ischemic reperfusion, HMGB1, inflammation cerebral infarct volume of brain tissue. The mechanisms underlying these effects may be associated with the inhibition of HMGB1 inflammatory signals.

\section{Introduction}

Ischemic stroke accounts for $75 \%$ of all stroke patients; it is a long-term disability and a leading cause of death worldwide (1). Thrombolytic and neuroprotective therapy are the major therapeutic strategies for ischemic stroke (2). Tissue-plasminogen activator (t-PA) is the only FDA-approved therapy for acute ischemic stroke, and must be used within a $3 \mathrm{~h}$ time window (3). Unfortunately, only $1-2 \%$ patients are able to receive thrombolytic therapy within this window. Around $60 \%$ stroke patients who receive intravenous tPA suffer fatality or become severely disabled (4). The poor efficiency of this treatment may be associated with the additional injury to the ischemic penumbra caused by reperfusion itself (I/R injury) (5). I/R injury is believed to aggravate cerebral injury through a series of inflammatory cascades, including the infiltration and accumulation of neutrophils and macrophages, the expression of certain cytokines, and the increased production of nitric oxide (6).

Traditional Chinese medicines are believed to be effective in treating patients with cerebral ischemia, and to have few clinical side-effects. Panax ginseng is a widely used medicinal herb, and its pharmacological effects have been previously demonstrated in various types of cancer, diabetes and cardiovascular diseases $(7,8)$. It is also commonly used for promoting immune function and central nervous system (CNS) function, and for its antioxidant activities (7). Ginsenosides are the major bioactive components of Panax ginseng, and are a group of saponins with a dammarane triterpenoid structure (8). Among these ginsenosides, Rb1 has been demonstrated to have protective effects on global cerebral I/R injury as well as acute myocardial ischemia in rats (9). However, the mechanism of the neuroprotective effect of $\mathrm{Rb} 1$ on focal cerebral I/R injury remains to be characterized. In the present study, we evaluated the influence of $\mathrm{Rb} 1$ on focal cerebral I/R injury usingthrough 
a MCAO-reperfusion model, and investigated the potential mechanisms underlying its protective effects.

\section{Materials and methods}

Experimental animals. A total of 50 Wistar rats (male; body weight, 270-330 g, age: 10 weeks-12 weeks) were obtained from the Animal Center of Shandong University (Jinan, China). All rats were maintained at $25 \pm 1^{\circ} \mathrm{C}$, with $12 \mathrm{~h}$ light $/ 12 \mathrm{~h}$ dark cycle of housing, food and water available. All animal experiment protocols were approved by the Institutional Animal Care Committee of Shandong University (Jinan, China), and were performed in strict consistence with its guidelines.

Ischemia-reperfusion model. After 1 week of accommodation, the rats were subjected to middle cerebral artery occlusion (MCAO) surgery as previously described $(10,11)$. Briefly, following anesthetization with $10 \%$ chloral hydrate $(350 \mathrm{mg} / \mathrm{kg}$; administered intraperitoneally) the left common carotid artery (CCA) was revealed and clipped using an artery clamp. During surgery, body temperature was maintained at $36.5-37.0^{\circ} \mathrm{C}$ using a heating pad on the surgical table. The incision region was disinfected with povidone-iodine solution. The external carotid artery (ECA) was separated and ligatured. A nylon suture with a blunted tip ( $0.35 \mathrm{~mm}$ diameter) was drawn into theECA and then into internal carotid artery (ICA). The middle cerebral artery was occluded by the suture $18 \mathrm{~mm}$ distal from the carotid bifurcation. Ischemia reperfusion injury was executed by removing the suture after $2 \mathrm{~h}$ of occlusion. Following closure of the incision, the rats were returned to cages with food and water available after the incision was closed.

Grouping and drug administration. Rb1 was dissolved in saline and intravenously injected following initiation of ischemia. The animals were randomized distributed into 5 groups according to the random number table. Firstly, the rats were numbered by body weight. Second, we chose the any row in the random number table and copy 50 random numbers. Sort random numbers from small to large. Specify the first 10 numbers as the first group from the sorted numbers, followed by analogy: i) sham control, the ECA was surgically prepared for insertion of the filament as described above, but the filament was not inserted and saline was received intravenously; ii) MCAO group, subjected to MCAO and saline was received intravenously and iii) Rb1 group, subjected to MCAO and 50,100 or $200 \mathrm{mg} / \mathrm{kg}$ of Rb1 was received intravenously. We conducted a pre-experiment to investigate the effect of $\mathrm{Rb} 1100 \mathrm{mg} / \mathrm{kg}$ on the focal cerebral ischemic reperfusion rats. The results manifested that $\mathrm{Rb} 100 \mathrm{mg} / \mathrm{kg}$ remarkably decreased the ischemic injury. Therefore, we chose Rb1 50, 100 and $200 \mathrm{mg} / \mathrm{kg}$ as the dose of Rb1.

Evaluation of neurological deficits. Neurological examination was performed blindly $24 \mathrm{~h}$ after reperfusion, according to Zea Longa's method (10). The scores of the neurological tests were categorized according to 5 grades: 0 , no neurological deficit; 1 , unable to extend right forepaw fully upon lifting of the whole body by the tail; 2 , circling to the right; 3 , falling to the right, and 4 , unable to walk spontaneously and reduced levels of consciousness.
Triphenyltetrazolium chloride (TTC) staining and infarct volume assessments. Coronal brain sections (2-mm thickness) were incubated with $2 \% \mathrm{TTC}$ at $37^{\circ} \mathrm{C}$ for 30 min with gentle agitation, then fixed with $10 \%$ formalin in PBS. Pale unstained sections were considered to be indicative of infarct regions, whereas red-stained sections were indicative of normal tissue. The slices were photographed from each side, and the infarct regions and were detected both hemispheres using a morphological image-analysis system (Jie Da software, China). Infarct volume was calculated as a percentage of the contralateral hemisphere volume using an 'indirect method' (area of intact contralateral hemisphere-area of intact regions of the ipsilateral hemisphere) to compensate for edema formation in the ipsilateral hemisphere. The volume of infarction was obtained according to the following formula, and expressed as percentage of infarction in the ipsilateral hemisphere (11):

$$
\begin{gathered}
V=\sum_{i=1}^{n-1} \frac{\left(A_{i}+A_{i+1}\right)}{2} \times h \\
V=\sum_{i=1}^{n-1} \frac{\left(A_{i}+A_{i+1}\right)}{2} \times h
\end{gathered}
$$

$\mathrm{V}$, volume of fraction; Ai, infarct area of each slice, and h, slice thickness.

Histological examination and TUNEL staining. After being anesthetized with $10 \%$ chloral hydrate $(350 \mathrm{mg} / \mathrm{kg}$; injected intraperitoneally) the rats were sacrificed by cardiac perfusion, the brains were immediately removed and the bregma-3 3.8 mm areas were immobilized in $4 \%$ neutral buffered formalin and embedded in paraffin. No peritonitis was observed in the rats during the entire experimental protocol. The areas were mounted onto slides, deparaffinized with xylene, rehydrated using a graded alcohol series, stained with hematoxylin and eosin and analyzed under a light microscope at magnification, $\mathrm{x} 100$. The brains were sliced into $10-\mu \mathrm{m}$ thick coronal sections at the level of the bregma. TUNEL staining was performed using an in situ apoptosis detection kit (Nanjing KeyGen Biotech Co., Ltd., Nanjing, China), according to the manufacturer's protocol. TUNEL staining was detected under a fluorescence microscope (Olympus IX71; Olympus Corporation, Tokyo, Japan). A total of 3 sections from each animal were analyzed by 2 investigators, blinded to the origin of the sections. For each section, TUNEL-positive cells were counted in 5 non-overlapping high-power fields at magnification, x200.

Western blotting analysis. Protein samples were prepared as previously described (12) and the protein concentration was determined using the Bradford method. The protein samples were heated at $95^{\circ} \mathrm{C}$ for $5 \mathrm{~min}$, loaded at $30 \mu \mathrm{g}$ per lane, separated using 10\% SDS-PAGE, and electrotransferred onto polyvinylidene difluoride membranes. The membranes were incubated with primary antibodies for cleaved caspase-3 (cat. no., 9664; Cell Signaling Technology, Inc., Danvers, MA, USA), cleaved caspase-9 (cat. no., 9507; Cell Signaling Technology, Inc.), with $\beta$-actin functioning as a loading control (cat. no., ab6276; Abcam, Cambridge, UK), overnight at $4^{\circ} \mathrm{C}$. Following washing with Tris-buffered saline with Tween 
(TBS-T), the membranes were incubated with a horseradish peroxidase-conjugated secondary antibody for $1 \mathrm{~h}$ at room temperature, then washed again with TBS-T. The antibodies were then visualized by enhanced chemiluminescence and the density of the protein bands was analyzed using an AlphaEaseFC system (ProteinSimple, San Jose, CA, USA).

ELISA. Cortex samples were homogenized in $1 \mathrm{ml}$ homogenization buffer and centrifuged at $14,000 \mathrm{x} g$ for $10 \mathrm{~min}$ at $4^{\circ} \mathrm{C}$. ELISA kits were used to verify the levels of high-mobility group box 1 (HMGB1) and NF- $\mathrm{B}$ p65, TNF- $\alpha$, iNOS, NO and IL-6, according to the manufacturer's instructions (Nanjing Jiancheng Bioengineering Institute, Nanjing, China).

Statistical analysis. All data are expressed as the mean \pm standard deviation and analyzed using one-way analysis of variance followed by the Least Significant Difference test. All the statistics analyses were performed using SPSS software (v.18; SPSS, Inc., Chicago, IL, USA). P<0.05 was considered to indicate a statistically significant difference.

\section{Results}

Rbl attenuates neurological deficits in MCAO animals. Neurological scores were determined $24 \mathrm{~h}$ after I/R injury. No neurological deficitobserved in sham animals, whereas MCAO animals suffered from I/R injury, displayed all the characteristics of neuron damage and had relatively high neurological deficit scores $(2.07 \pm 0.24$; Table I). The results also show that Rb1 treatment significantly improved the neurological deficits of MCAO mice, and the deficit score in animals treated with 50,100 and $200 \mathrm{mg} / \mathrm{kg} \mathrm{Rb1}$ were decreased to $1.71 \pm 0.43$, $1.25 \pm 0.72$ and $1.05 \pm 0.36$, respectively.

Rbl reduces cerebral infarct volume in the MCAO rat model. Infarct area of brain tissues from the animals measured $24 \mathrm{~h}$ after I/R injury by TTC staining are presented in Fig. 1. No infarct was observed in sham animals, whereas in the MCAO group, the infarct area reached $31.56 \%$ the whole brain. However, as shown in Fig. 1B, Rb1-treatment decreased infarct volumes in MCAO rats in a dose-dependent manner: 50, 100 and $200 \mathrm{mg} / \mathrm{kg}$ $\mathrm{Rb} 1$ treatment reduced the infarct volume to $25.89 \%$ ( $\mathrm{P}<0.05$ vs. MCAO animals), $18.35 \%$ ( $\mathrm{P}<0.01$ vs. MCAO animals) and $10.13 \%$ ( $\mathrm{P}<0.01$ vs. MCAO animals), respectively.

Rbl treatment improves brain histopathological abnormalities and neuron apoptosis. Hematoxylin and eosin staining was applied to examine the histopathological abnormalities following focal cerebral I/R (Fig. 2). No histopathological damage was detected in the corext or pyramidal neurons in the hippocampus CA1 region of sham animals (Fig. 2A and B). In the MCAO rat model, the majority of the neurons in the infarct core were atrophied and/or reduced in size, exhibiting a eosinophilic cytoplasm and triangulated pycnotic nucleus compared with the intact and well-arranged neurons with eumorphism in the sham group. However, the number of pyramidal neurons in the MCAO model was significantly decreased compared with the sham group, and large necrotic neurons surrounding the infarct core and in the peri-infarct zone were noted, exhibiting pycnotic shape and condensed nuclear material. The necrotic
Table I. Effects of Rb1 on neurological deficit scores in rats $24 \mathrm{~h}$ after reperfusion.

\begin{tabular}{lcc}
\hline Groups & Rat no. (n) & Neurological scores \\
\hline Sham & 8 & $0.00 \pm 0.00^{\mathrm{b}}$ \\
MCAO & 8 & $2.07 \pm 0.24$ \\
Rb1 $50 \mathrm{mg} / \mathrm{kg}$ & 8 & $1.71 \pm 0.43$ \\
Rb1 $100 \mathrm{mg} / \mathrm{kg}$ & 8 & $1.25 \pm 0.72^{\mathrm{a}}$ \\
Rb1 $200 \mathrm{mg} / \mathrm{kg}$ & 8 & $1.05 \pm 0.36^{\mathrm{b}}$ \\
\hline
\end{tabular}

Data are presented as the mean \pm standard deviation. Animals received different doses of $\mathrm{Rb} 1$ or saline vehicle following the onset of ischemia. Neurological deficit scores were evaluated after $24 \mathrm{~h}$ of reperfusion according to the Longa's method. ${ }^{\mathrm{a}} \mathrm{P}<0.05,{ }^{\mathrm{b}} \mathrm{P}<0.01$ vs. MCAO. RB1, Ginsenoside Rb1; MCAO, middle cerebral artery occlusion.

tissue was notablyremarkably diminished following Rb1 treatment. This suggests a reduction in nerve injury, characterized by the decreased number of cells with obvious historical change, such as liquefaction necrosis, pycnosis, nucleoli abolition and nuclear fragmentation.

Adjusted expression of HMGBl and inflammatory factors by $R b 1$. The level of HMGB1 in the peri-infarct zones of ischemic cortex samples from each group was measured. A significantly increased level of HMGB1 was identified in brain tissue subjected to focal cerebral ischemia reperfusion. Furthermore, an increased level of HMGB1 was observed in MCAO rats compared with the sham group $(\mathrm{P}<0.01$; Fig. $3 \mathrm{~A})$, which was notably decreased by Rb1-treatment.

High levels of TNF- $\alpha$, iNOS, NO and IL- 6 were also identified in MCAO rats $(\mathrm{P}<0.05$, sham vs. MACO; Fig. 3), which was significantly attenuated in Rb1-treated animals.

Decrease of neuronal cell apoptosis by Rbl treatment. Neuronal cell apoptosis was measured by TUNEL staining in the rats of each group (Fig. 4). The number of TUNEL-positive neurons in the cortex region was significantly increased in MCAO group after I/R injury compared with the sham group $(\mathrm{P}<0.01)$. Following $\mathrm{Rb} 1$ treatment, the number of TUNEL-positive neurons in the cortex region was significantly reduced compared with the sham group ( $\mathrm{P}<0.05$; Fig. 4$)$. A higher proportion of apoptotic neurons was detected in MCAO group, compared with the sham group $(\mathrm{P}<0.01)$. Rb1 treatment at 50,100 or $200 \mathrm{mg} / \mathrm{kg}$ reduced the TUNEL-positive staining by $30.2,19.6$ and $9.2 \%$, respectively ( $\mathrm{P}<0.01$ vs. MCAO).

The expression levels of cleaved caspase- 3 and caspase- 9 was also investigated. High expression of apoptosis-related proteins was exhibited by brain tissues from the MCAO group compared with the sham group $(\mathrm{P}<0.05$; Fig. 4D). The elevated levels of cleaved caspase- 3 and caspase- 9 were significantly attenuated with Rb1 treatment (Fig. 4C and D).

\section{Discussion}

Natural products can be used to modulate cytokine-activity for treatment of diseases (5). In the present study, Rb1, one of 


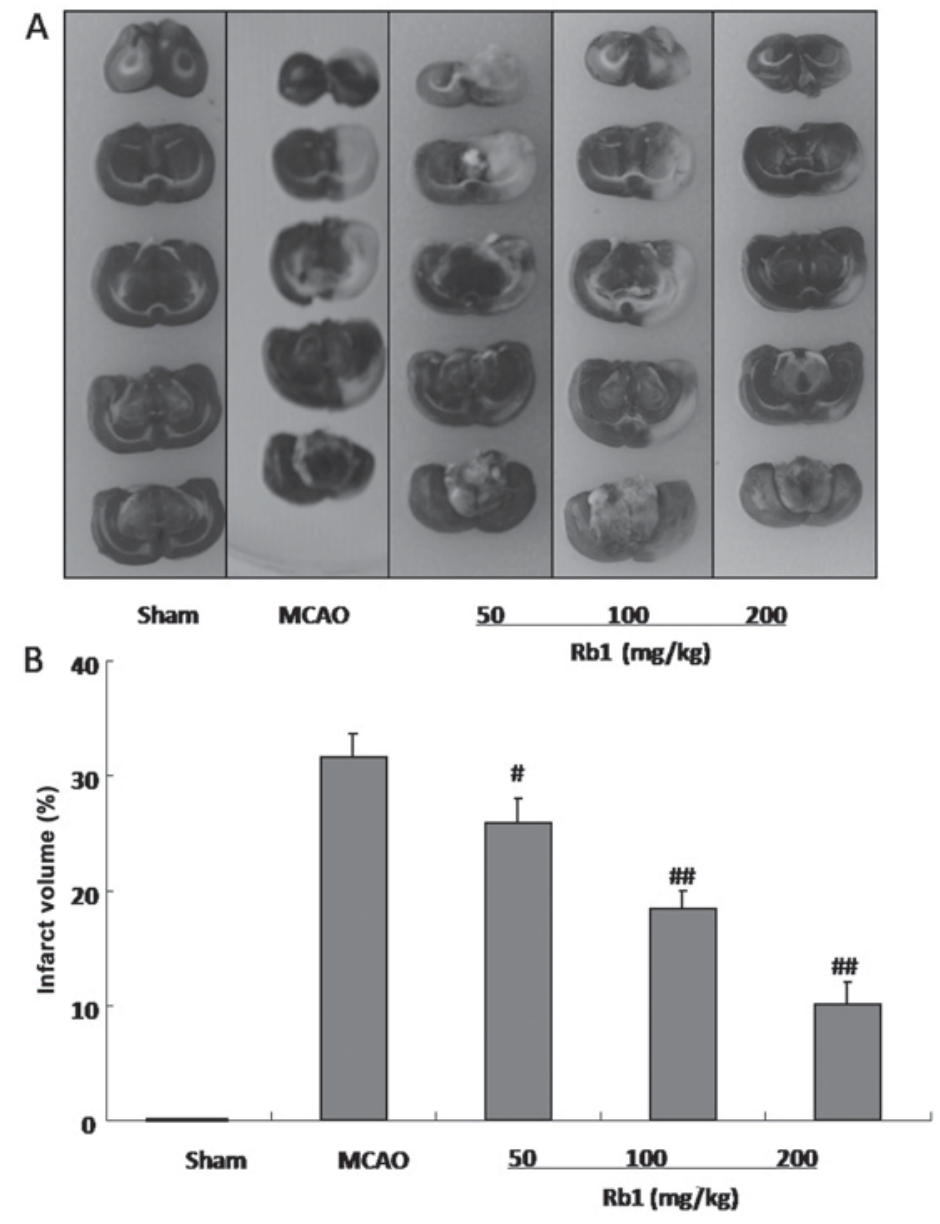

Figure 1. Effect of Rb1 on cerebral infarct area in MCAO rats. (A) Cerebral infarct area stained with TTC in different groups. The coronal sections were obtained after $24 \mathrm{~h}$ of reperfusion. (B) Evaluation of infarct area after $24 \mathrm{~h}$ of reperfusion, the bar indicates the percentage of infarct area. Data are expressed as the mean \pm standard deviation $(\mathrm{n}=8) .{ }^{\#} \mathrm{P}<0.05,{ }^{\# \#} \mathrm{P}<0.01,{ }^{\# \#} \mathrm{P}<0.001$ vs. $\mathrm{MCAO}$ group. MCAO, middle cerebral artery occlusion; TTC, Triphenyltetrazolium chloride.
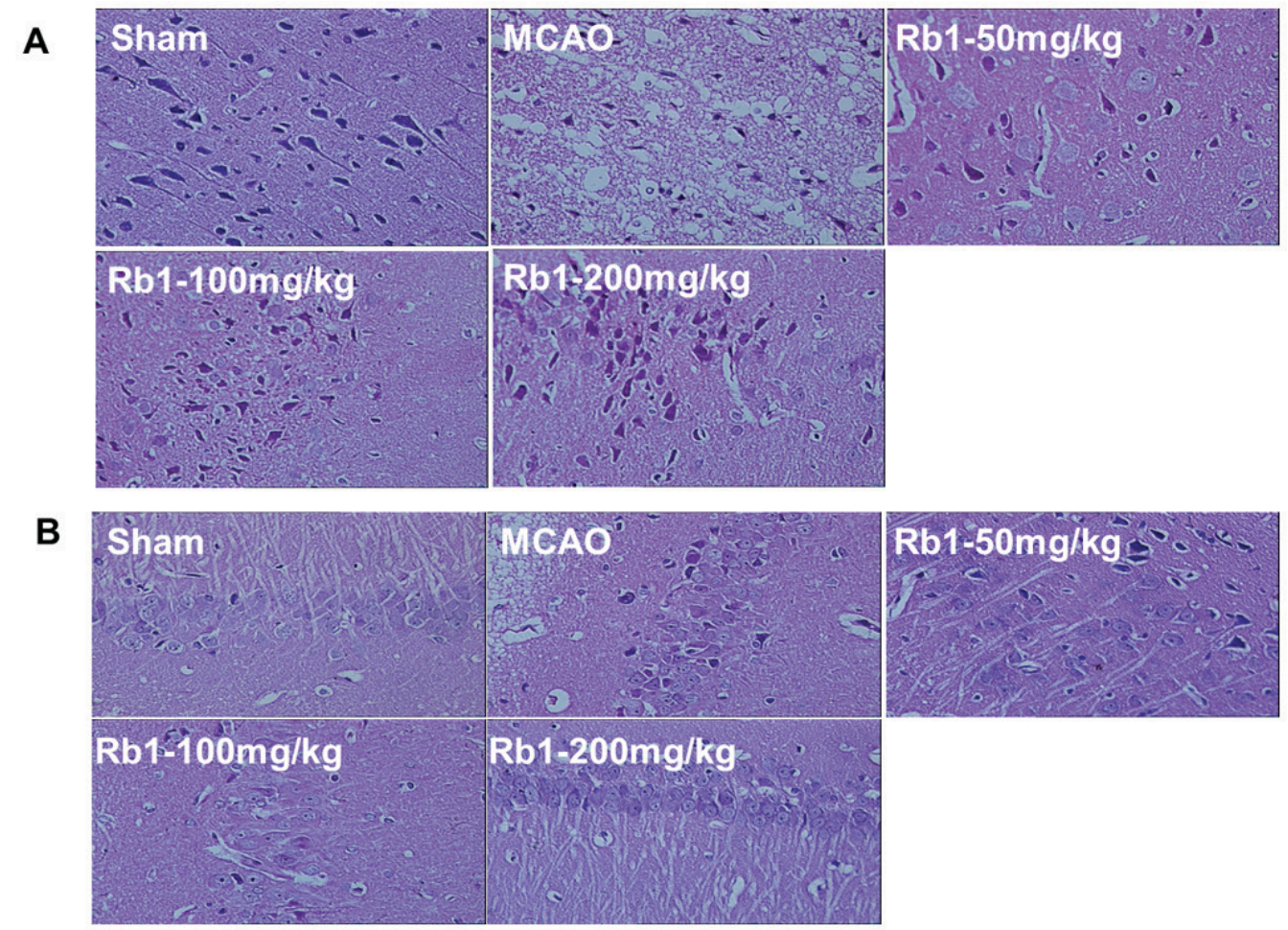

Figure 2. Histopathology of the cortical and hippocampal CA1 neurons of rats examined by Hematoxylin and eosin staining. Representative micrographs of the (A) cortex and (B) hippocampus of the sham, MCAO and Rb1 treated groups, respectively. Magnification, x100. MCAO, middle cerebral artery occlusion. 

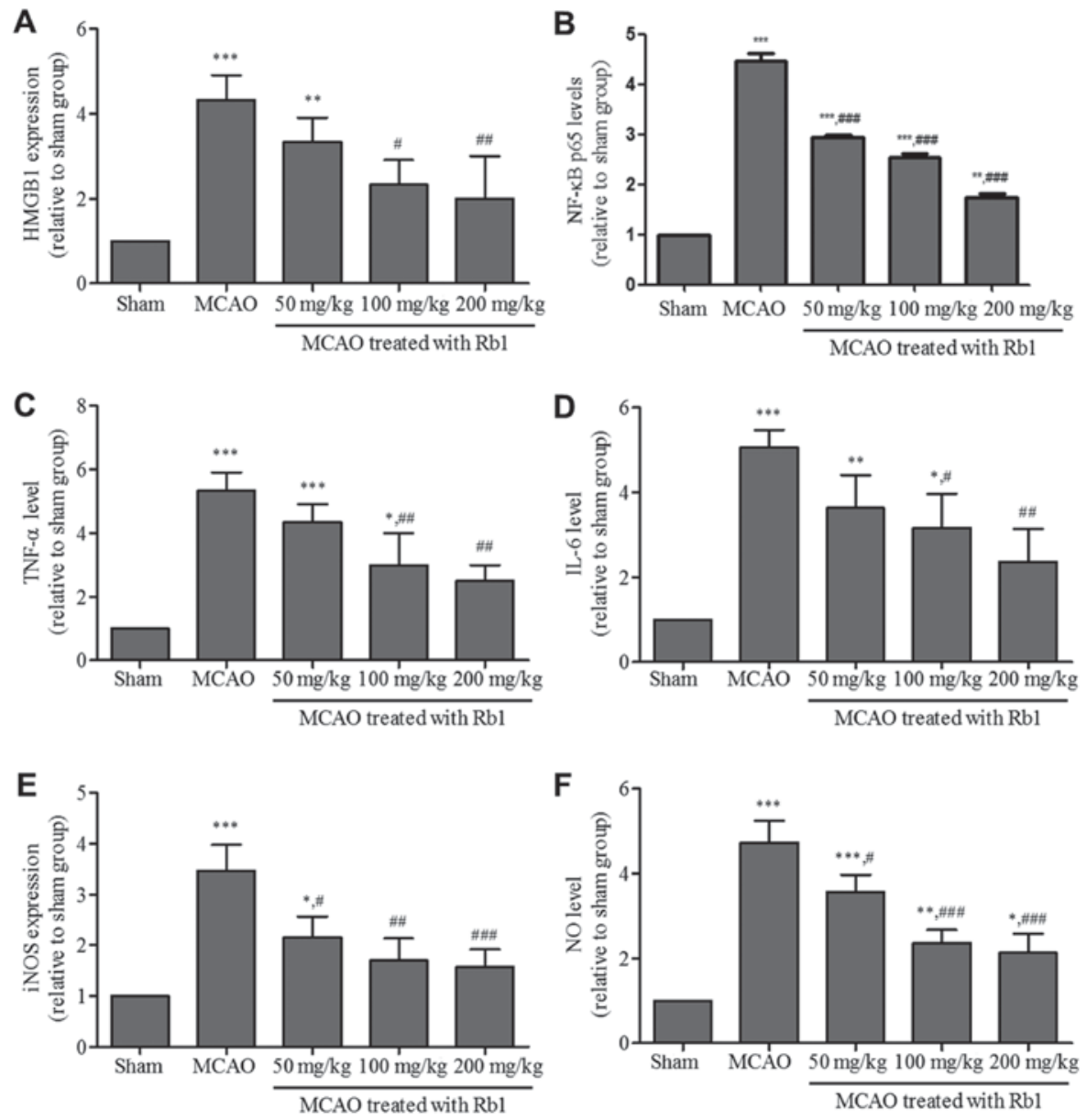

Figure 3. Analysis of the levels of HMGB1 and inflammatory factors. (A) HMGB1 levels, measured by ELISA. (B) NF- $\kappa$ B expression levels. Levels of pro-inflammation factors, (C) TNF- $\alpha$, (D) IL-6, (E) iNOS and (F) NO measured by ELISA. ${ }^{*} \mathrm{P}<0.05,{ }^{* *} \mathrm{P}<0.01,{ }^{* * *} \mathrm{P}<0.001$ vs. sham group; ${ }^{*} \mathrm{P}<0.05,{ }^{\# \#} \mathrm{P}<0.01$,

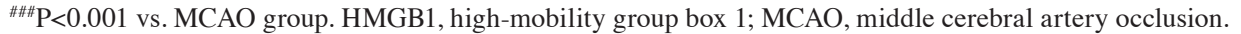

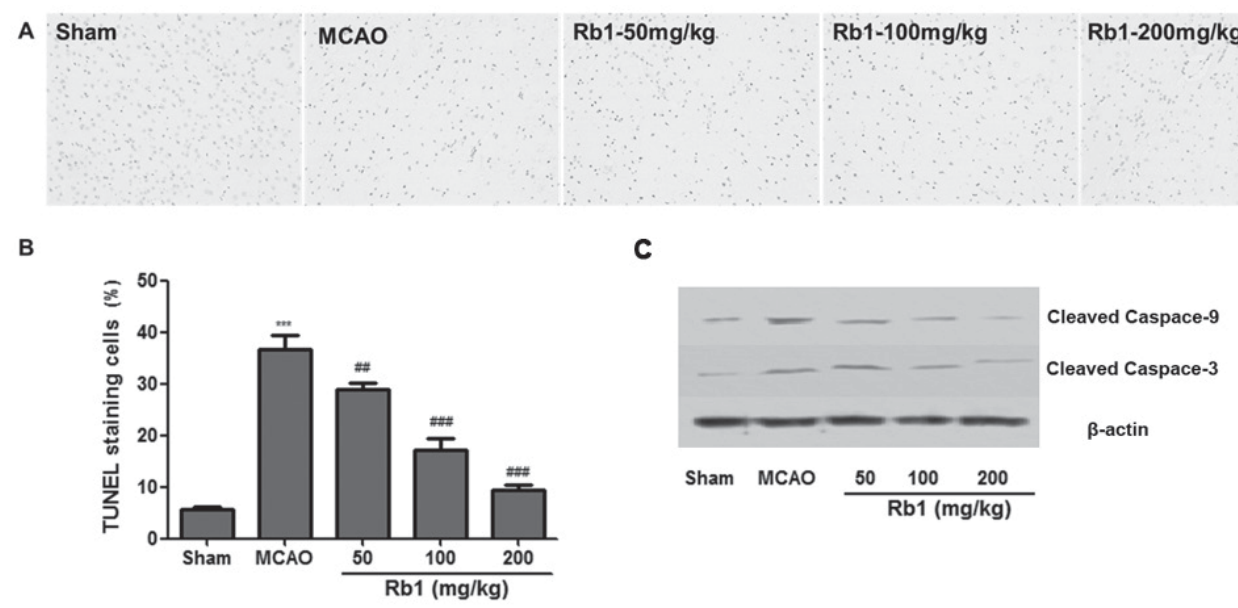

Figure 4. Rb1 protects neuronal cells from cerebral ischemic reperfusion-induced apoptosis in the cortex. (A) Cell apoptosis in cortex tissue measured by TUNEL staining. Magnification, $\mathrm{x} 200$. (B) Analysis of the percentage of TUNEL-positive cells. ${ }^{* * *} \mathrm{P}<0.001$ vs. sham group; ${ }^{\# \#} \mathrm{P}<0.01$ and ${ }^{\# \# \#} \mathrm{P}<0.001 \mathrm{vs}$. MCAO group. (C) Protein expression analyzed by western blotting.

the major bioactive components of Panax ginseng, improved the neurological function and decreased the infarct volume in brain tissues of a MCAO rat model. HMGB1 levels in the brain tissue were significantly decreased in MCAO rats after $\mathrm{Rb} 1$ treatment, and the levels HMGB1-associated inflammatory factors, including TNF- $\alpha$, IL-6, iNOS and NO, were also reduced following Rb1 treatment. These data suggested that Rb1 may have a neuroprotective effect against I/R injury during stroke therapy.

Numerous studies have demonstrated the beneficial effects of $\mathrm{Rb} 1$ in the treatment of ischemic stroke $(9,13-15)$, however, further research is required to understand the mechanisms of 
Rb1 function. An inflammatory reaction in the brain causes ischemic stroke, which occurs in $\sim 80 \%$ stroke patients, and causes the release of free radicals, resulting in oxidative damage of brain tissues $(13,14)$. High mobility group box 1 (HMGB1) is a highly conserved non-histone DNA-binding nuclear protein, and a well-known damage-associated molecular pattern molecule which can promote inflammatory injury. It is well established that HMGB1-mediated inflammation may be a cause of cerebral I/R-induced brain damage (16). Previous research has demonstrated that inhibition of HMGB1 is associated with suppression of infarct formation $(17,18)$. Other studies have implied that $\mathrm{NF}-\kappa \mathrm{B}$ may be a key regulator of inflammation during and subsequent to brain damage $(19,20)$. It has been suggested that HMGB1 could rapidly bind to TLR-2 and inhibit its expression, subsequently blocking NF-KB activation induced by HMGB1 (21). In the present study, significantly increased HMGB1 levels accompanied by increased NF-KB levels in the MCAO model were demonstrated, which is consistent with these previous reports. It was also demonstrated that Rb1 administration markedly reduced the elevated HMGB1 and NF- $\kappa \mathrm{B}$ levels in the MCAO model. Therefore, we hypothesized that Rb1 treatment may lead to the downregulation of HMGB1 signaling and that downstream molecules activate $\mathrm{NF}-\kappa \mathrm{B}$ p65, consequently attenuating the I/R injury. Previous reports have demonstrated that HMGB1 could upregulate the levels of TNF- $\alpha$ and IL-6, which can also promote HMGB1 release via positive-feedback $(22,23)$. Indicators of inflammation were also identified in the present study in the cerebral I/R, including TNF- $\alpha$, IL- 6 and iNOS, whose levels were significantly decreased in the cortex tissue of MCAO rats.

Furthermore, HMGB1 is a crucial proinflammatory factor in ischemic stroke and the signal is transduced via its putative receptors, such as toll-like receptors (TLRs), receptor for advanced glycation end products (RAGE) and matrix metalloproteinase (MMP) enzymes during ischemic stroke. The present study suggests that Rb1 administration could markedly reduce the elevated levels of HMGB1 and $\mathrm{NF}-\kappa \mathrm{B}$ in MCAO rats. However, the effects of Rb1 on HMGB1-associated receptors remain to be elucidated. HMGB1 may be a novel subject of brain-immune communication and post-stroke immunomodulation research. In acute ischemic stroke patients, the peripheral percentage of some subsets of T-lymphocytes was associated with the level of neurological deficit, and a predictive role of the peripheral percentage of CD28-null cells in stroke diagnosis and TOAST subtyping was suggested $(24,25)$. Therefore, HMGB1 is a promising therapeutic in promoting neurovascular repair and remodeling following stroke.

$\mathrm{I} / \mathrm{R}$ injury-induced oxidative stress and inflammation also triggers multiple-cell apoptotic pathways responsible for cell death by necrosis or apoptosis (26-28). Previous research has demonstrated that HMGB1 could promote the apoptosis of myocytes $(22,29)$. In both MCAO rat models and stroke patients, persistent $\mathrm{NF}-\kappa \mathrm{B}$ p65 activation has been indicated to contribute to infarction and cell death induced by the I/R injury $(6,30)$. In the present study, Rb1 treatment decreased neuronal cell apoptosis in MCAO rats in a dose-dependent manner. Bcl-2 and Bax, belonging to the Bcl-2 family, are key physiological and pathological regulators of cell apoptosis, and act via the activation of caspase-triggered signaling cascades $(31,32)$. The present study demonstrated that Rb1 markedly downregulated the levels of caspase- 3 and caspase-9, indicating that Rb1 suppressed cerebral I/R-induced cell apoptosis in the brain tissue of MCAO rats by mediating caspase-3-associated proteins.

In summary, the present study demonstrated that Rb1 has a protective effect on cerebral neurons in I/R injury. The mechanisms underlying these actions are not well established, however, our results suggest that the inhibition of inflammatory HMGB1 signaling may serve an important role in the process. Furthermore, Rb1 may be a promising neuroprotective candidate, and requires further laboratory and clinical investigation.

\section{Acknowledgements}

The authors would like to thank the Central Research Laboratory, The Second Hospital of Shandong University for technical assistance and the generous support.

\section{Funding}

The present study was supported by the Natural Science Foundation of China (grant no. 81402962).

\section{Availability of data and materials}

All of the materials used in the present study are commercially available and all data included in the present study were obtained by the co-authors.

\section{Authors' contributions}

AL, GH, ZC and LZ designed the study. HL, XX and WJ performed the experiments. WZ, LS analyzed the data. HL and $\mathrm{XX}$ wrote the manuscript.

\section{Ethics approval and consent to participate}

All animal experiment protocols were approved by the Institutional Animal Care Committee of Shandong University (Jinan, China), and were performed in strict consistence with its guidelines.

\section{Patient consent for publication}

Not applicable.

\section{Competing interests}

The authors declare that they have no competing interests.

\section{References}

1. Zevallos J, Santiago F, González J, Rodríguez A, Pericchi L, Rodríguez-Mercado R and Nobo U: Burden of stroke in puerto rico. Int J Stroke 10: 117-119, 2015.

2. Shi GD, OuYang YP, Shi JG, Liu Y, Yuan W and Jia LS: PTEN deletion prevents ischemic brain injury by activating the mTOR signaling pathway. Biochem Biophys Res Commun 404: 941-945, 2011. 
3. Cronin CA: Intravenous tissue plasminogen activator for stroke: a review of the ECASS III results in relation to prior clinical trials. J Emerg Med 38: 99-105, 2010.

4. Kirmani JF, Alkawi A, Panezai S and Gizzi M: Advances in thrombolytics for treatment of acute ischemic stroke. Neurology 79: S119-S125, 2012.

5. Sun K, Fan J and Han J: Ameliorating effects of traditional Chinese medicine preparation, Chinese materia medica and active compounds on ischemia/reperfusion-induced cerebral microcirculatory disturbances and neuron damage. Acta Pharm Sin B 5: 8-24, 2015.

6. Xue X, Qu XJ, Yang Y, Sheng XH, Cheng F, Jiang EN, Wang JH, Bu W and Liu ZP: Baicalin attenuates focal cerebral ischemic reperfusion injury through inhibition of nuclear factor $\kappa \mathrm{B}$ p65 activation. Biochem Biophys Res Commun 403: 398-404, 2010.

7. Jung NP and Jin SH: Studies on the physiological and biochemical effect of Korean ginseng. Korean J Ginseng Sci 20: 431-471, 1996.

8. Li C, Zhu Y, Guo X, Sun C, Luo H, Song J, Li Y, Wang L, Qian J and Chen S: Transcriptome analysis reveals ginsenosides biosynthetic genes, microRNAs and simple sequence repeats in Panax ginseng C. A. Meyer. BMC Genomics 14: 245, 2013.

9. Zhang J, Han X, Li X, Luo Y, Zhao H, Yang M, Ni B and Liao Z: Core-shell hybrid liposomal vesicles loaded with panax notoginsenoside: Preparation, characterization and protective effects on global cerebral ischemia/reperfusion injury and acute myocardial ischemia in rats. Int J Nanomedicine 7: 4299-4310, 2012.

10. Longa EZ, Weinstein PR, Carlson S and Cummins R: Reversible middle cerebral artery occlusion without craniectomy in rats. Stroke 20: 84-91, 1989.

11. Wei X, Liu H, Sun X, Fu F, Zhang X, Wang J, An J and Ding H: Hydroxysafflor yellow A protects rat brains against ischemia-reperfusion injury by antioxidant action. Neurosci Lett 386: 58-62, 2005

12. Okuno S, Saito A, Hayashi T and Chan PH: The c-Jun N-terminal protein kinase signaling pathway mediates Bax activation and subsequent neuronal apoptosis through interaction with Bim after transient focal cerebral ischemia. J Neurosci 24: 7879-7887, 2004

13. Park EK, Choo MK, Oh JK, Ryu JH and Kim DH: Ginsenoside Rh2 reduces ischemic brain injury in rats. Biol Pharm Bull 27: 433-436, 2004

14. Huang XP, Qiu YY, Wang B, Ding H, Tang YH, Zeng R and Deng CQ: Effects of Astragaloside IV combined with the active components of Panax notoginseng on oxidative stress injury and nuclear factor-erythroid 2-related factor $2 /$ heme oxygenase-1 signaling pathway after cerebral ischemia-reperfusion in mice. Pharmacogn Mag 10: 402-409, 2014.

15. Ye R, Kong X, Yang Q, Zhang Y, Han J, Li P, Xiong L and Zhao G: Ginsenoside rd in experimental stroke: superior neuroprotective efficacy with a wide therapeutic window. Neurotherapeutics 8: 515-525, 2011.

16. Zheng C, Liu C, Wang W, Tang G, Dong L, Zhou J and Zhong Z: Ethanol extracts from Portulaca oleracea L. attenuated ischemia/reperfusion induced rat neural injury through inhibition of HMGB1 induced inflammation. Am J Transl Res 8: 5016-5024, 2016.

17. Jin YC, Kim SW, Cheng F, Shin JH, Park JK, Lee S, Lee JE, Han PL, Lee M, Kim KK, et al: The effect of biodegradable gelatin microspheres on the neuroprotective effects of high mobility group box $1 \mathrm{~A}$ box in the postischemic brain. Biomaterials 32: 899-908, 2011.

18. Kim SW, Jin Y, Shin JH, Kim ID, Lee HK, Park S, Han PL and Lee JK: Glycyrrhizic acid affords robust neuroprotection in the postischemic brain via anti-inflammatory effect by inhibiting HMGB1 phosphorylation and secretion. Neurobiol Dis 46 : $147-156,2012$
19. Kim JW, Jin YC, Kim YM, Rhie S, Kim HJ, Seo HG, Lee JH, Ha YL and Chang KC: Daidzein administration in vivo reduces myocardial injury in a rat ischemia/reperfusion model by inhibiting NF-kappaB activation, Life Sci 84: 227-234, 2009.

20. Yang L, Tao LY and Chen XP: Roles of NF-kappaB in central nervous system damage and repair, Neurosci. Bull 23: 307-313, 2007

21. Park JS, Svetkauskaite D, He Q, Kim JY, Strassheim D, Ishizaka A and Abraham E: Involvement of toll-like receptors 2 and 4 in cellular activation by high mobility group box 1 protein. J Biol Chem 279: 7370-7377, 2004.

22. Li X, Hu X, Wang J, Xu W, Yi C, Ma R and Jiang H: Short-term hesperidin pretreatment attenuates rat myocardial ischemia/reperfusion injury by inhibiting high mobility group box 1 protein expression via the PI3K/Akt pathway. Cell Physiol Biochem 39: 1850-1862, 2016.

23. Andersson U, Wang H, Palmblad K, Aveberger AC, Bloom O, Erlandsson-Harris H, Janson A, Kokkola R, Zhang M, Yang H and Tracey KJ: High mobility group 1 protein (HMG-1) stimulates proinflammatory cytokine synthesis in human monocytes. J Exp Med 192: 565-570, 2000.

24. ATuttolomondo A, Pecoraro R, Casuccio A, Di Raimondo D, Butt Ã C, Clemente G, Della Corte V, Guggino G, Arnao V, Maida C, et al: Peripheral frequency of CD4+CD28-cells in acute ischemic stroke: Relationship with stroke subtype and severity markers. Medicine (Baltimore) 94: e81, 2015.

25. Tuttolomondo A, Pedone C, Pinto A, Di Raimondo D, Fernandez P, Di Sciacca R and Licata G; Gruppo Italiano di Farmacoepidemiologia dell'Anziano (GIFA) researchers. Predictors of outcome in acute ischemic cerebrovascular syndromes: The GIFA study. Int J Cardiol 125: 391-396, 2008.

26. Hu GQ, Du X, Li YJ, Gao XQ, Chen BQ and Yu L: Inhibition of cerebral ischemia/reperfusion injury-induced apoptosis: Nicotiflorin and JAK2/STAT3 pathway. Neural Regen Res 12: 96-102, 2017.

27. Li P, Nijhawan D, Budihardjo I, Srinivasula SM, Ahmad M, Alnemri ES and Wang X: Cytochrome $\mathrm{c}$ and dATP-dependent formation of Apaf-1/caspase-9 complex initiates an apoptotic protease cascade. Cell 91: 479-489, 1997.

28. Polster BM and Fiskum G: Mitochondrial mechanisms of neural cell apoptosis. J Neurochem 90: 1281-1289, 2004.

29. Zhang HL, Gu ZL, Savitz SI, Han F, Fukunaga K and Qin ZH: Neuroprotective effects of prostaglandin A(1) in rat models of permanent focal cerebral ischemia are associated with nuclear factor-kappaB inhibition and peroxisome proliferator-activated receptor-gamma up-regulation. J Neurosci Res 86: 1132-1141, 2008.

30. Hu X, Cui B, Zhou X, Xu C, Lu Z and Jiang H: Ethyl pyruvate reduces myocardial ischemia and reperfusion injury by inhibiting high mobility group box 1 protein in rats. Mol Biol Rep 39: 227-231, 2012.

31. Nurmi A, Lindsberg PJ, Koistinaho M, Zhang W, Juettler E, Karjalainen-Lindsberg ML, Weih F, Frank N, Schwaninger M and Koistinaho J: Nuclear factor-kappaB contributes to infarction after permanent focal ischemia. Stroke 35: 987-991, 2004.

32. Zhou JQ, Qiu T, Zhang L, Chen ZB, Wang ZS, Ma XX and Li D: Allopurinol preconditioning attenuates renal ischemia/reperfusion injury by inhibiting HMGB1 expression in a rat model. Acta CBras 31: 176-182, 2016.

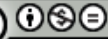

This work is licensed under a Creative Commons Attribution-NonCommercial-NoDerivatives 4.0 International (CC BY-NC-ND 4.0) License. 